\title{
A Study on the Law of Birth and Formation of Yang-Deficiency Constitution Based on the Theory of Five Movement and Six Qi
}

\author{
Jingyi Bai', Xinli Wen ${ }^{2 *}$ \\ ${ }^{1}$ Shaanxi University of Traditional Chinese Medicine, Xianyang 712046, Shaanxi Province, China \\ ${ }^{2}$ Shaanxi Provincial Hospital of Traditional Chinese Medicine, Xi'an 710000, Shaanxi Province, China \\ *Corresponding author: Xinli Wen, jinyib01@163.com
}

\begin{abstract}
Objective: To explore the law of birth and yang deficiency constitution based on the theory of five movements and six qi, as well as to provide a new way to better realize the prevention of yang deficiency constitution. Methods: The data of TCM quality table and luck data were collected via questionnaires. According to the data of from the TCM quality table, the respondents were divided into yang deficiency constitution group and non-yang deficiency group. According to the luck data, the distributions of gestational age were compared between the two groups. Results: A total of 175 questionnaires were collected, of which 89 were non-yang deficiency constitution and 86 were yang deficiency constitution. The proportion of Ding year $(15.1 \%)$ in the yang deficiency group was significantly higher than that in non-yang deficiency group (5.6\%), in which the difference was statistically significant $(\mathrm{P}<0.05)$. Conclusion: The possibility of yang deficiency constitution is higher.
\end{abstract}

Keywords: Five movement six qi; Yang deficiency constitution; Gestational period; Year transport; Prevention before illness

Publication date: September 2021; Online publication: September 30, 2021

\section{Introduction}

It is known that the concept of "not ill" has been discussed in Huangdi Neijing. The "not ill" state in traditional Chinese medicine (TCM) includes sub-health states in modern medicine. Therefore, the concept of treating "not ill" in TCM can be used in the prevention and treatment of people with sub-optimal health ${ }^{[1]}$. According to the 2017 National Physique Identification Report, yang deficiency constitution accounted for the largest proportion of biased constitution among sub-healthy people, up to $16.40 \%$, and is closely related to IgA nephropathy, dysmenorrhea, and chronic fatigue syndrome ${ }^{[2]}$.

The theory of five movements and six qi discusses the periodic law of natural change and its influence on human health and diseases. The influence of luck on innate constitution is regarded as the effect on the pregnancy of the fetus and the tendency of acquired diseases ${ }^{[3]}$. This study is based on the theory of five movements and six qi to explore the law of birth and Yang deficiency constitution in order to give full play to the advantages of traditional Chinese medicine in treating "diseases without illnesses" and to provide a new idea for the prevention of yang deficiency constitution.

\section{Methods}

\subsection{Source of information}

The data of 175 people in Shaanxi were collected via questionnaires, including TCM quality table and luck 
data. The inclusion criteria were people who were able to complete the questionnaire independently.

\subsection{Methodology}

According to the Classification and Judgment of TCM Physique ${ }^{[4]}$, the results of the calculation were checked by two people to avoid errors of judgment due to calculation errors. According to the date of birth, the Gregorian calendar was used to determine the main days of pregnancy.

\subsection{Statistical methods}

The data were analyzed using Statistical Package for the Social Sciences (SPSS) version 26.0 software. P $<0.05$ was considered statistically significant.

\section{Results}

\subsection{Gender distribution analysis}

The proportions of males and females in the yang deficiency group were $31.4 \%$ and $68.6 \%$, respectively whereas in the non-yang deficiency group, the proportions were $48.3 \%$ and $51.7 \%$, respectively $(\mathrm{P}<0.05)$.

Table 1. Gender distribution

\begin{tabular}{cccccc}
\hline Gender & Yang deficiency & Non-yang deficiency & Total & $\chi^{2}$ value & P value \\
\hline Male & $27(31.4 \%)$ & $43(48.3 \%)$ & 70 & \multirow{2}{*}{5.217} & \multirow{2}{*}{0.022} \\
Female & $59(68.6 \%)$ & $46(51.7 \%)$ & 105 & & \\
\hline
\end{tabular}

\subsection{Analysis of the age transport subtypes of the two groups}

Among the yang deficiency group, wood transport was the most (15.1\%), soil transport was the least (2.3\%), whereas among the non-yang deficiency group, gold transport was the most (19.2\%), soil transport and fire transport was the least $(4.5 \%)$. The distribution difference was statistically significant $(\mathrm{P}<0.05)$.

Table 2. Analysis of the subtypes

\begin{tabular}{lccccc}
\hline Year of transport & Yang deficiency & Non-yang deficiency & Total & $\chi^{2}$ value & P value \\
\hline Excessive wood & $10(11.6 \%)$ & $11(12.4 \%)$ & 21 & 0.022 & 0.882 \\
Inadequate wood & $13(15.1 \%)$ & $5(5.6 \%)$ & 18 & 4.276 & 0.039 \\
Excessive fire & $6(7.0 \%)$ & $4(4.5 \%)$ & 10 & 0.500 & 0.479 \\
Inadequate fire & $8(9.3 \%)$ & $5(5.6 \%)$ & 13 & 0.863 & 0.353 \\
Excessive soil & $7(8.1 \%)$ & $9(10.1 \%)$ & 16 & 0.205 & 0.651 \\
Inadequate soil & $4(2.3 \%)$ & $4(4.5 \%)$ & 8 & 0.002 & 0.960 \\
Excessive gold & $11(12.8 \%)$ & $19(19.2 \%)$ & 30 & 1.388 & 0.239 \\
Inadequate gold & $10(11.6 \%)$ & $13(14.6 \%)$ & 23 & 0.340 & 0.560 \\
Excessive water & $7(8.1 \%)$ & $7(10 \%)$ & 14 & 0.004 & 0.947 \\
Inadequate water & $10(11.6 \%)$ & $12(13.5 \%)$ & 22 & 0.137 & 0.711 \\
\hline
\end{tabular}

\section{Discussion}

Yang deficiency constitution is the physical congenital state of the human body due to the deficiency of yang qi. According to the constitution theory in traditional Chinese medicine, the body and disease are 
related. Constitution determines the tendency of a type of disease, and it is an important factor that determines the nature, status, course, and trend of a disease ${ }^{[5]}$.

Modern studies have found that the essence of sub-health is the imbalance of yin and yang caused by the deficiency of yang qi in the body ${ }^{[6]}$. The study found that yang deficiency constitution is closely related to tumors ${ }^{[7,8]}$, elderly hypertension ${ }^{[9,10]}$, diabetes ${ }^{[11]}$, bronchial asthma ${ }^{[12,13]}$, osteoporosis ${ }^{[14,15]}$, infertility ${ }^{[16]}$, and so on. Therefore, it is of great significance to study the formation of yang deficiency constitution in regulating sub-health states.

Based on the theory of five transport and six qi, this study included Yang deficiency constitution and non-Yang deficiency constitution for comparative study. Through comparison, it was found that the possibility of Yang deficiency constitution formation increased in the year of Ding (lack of wood transport). In Huangdi Neijing, it has been mentioned, "It is not as good as, do be, threat and take." According to heavenly holistic thoughts, the human body easily forms yang deficiency constitution. The above suggests that theory and research coincide.

There are still shortcomings in this study, thus further studies with larger sample size is encouraged to provide further clinical evidence for the prevention and treatment of yang deficiency constitution.

\section{Disclosure statement}

The author declares no conflict of interest.

\section{References}

[1] Li L, Xue XL, Xu WJ, et al., 2008, Application of the Idea of "Treating Disease Without Disease" in the Prevention and Treatment of Subhealth. Beijing Traditional Chinese Medicine, 01: 76-77.

[2] Yan S, 2017, Yang Deficiency Constitution is the Most in Sub-Health People. Care Times.

[3] Xue LF, 2010, Clinical Investigation and Study on the Influence of Gasification Law on Children's Innate Constitution. Journal of Shandong University of Traditional Chinese Medicine, 3: 223-225.

[4] Chinese Society of Traditional Chinese Medicine, 2009, Classification and Judgment of TCM Physique, China Traditional Chinese Medicine Press, China, 303-304.

[5] Wang Q, 1995, Traditional Chinese Medical Constitution, China Medical Science and Technology Press, China.

[6] Xu ZH, 2009, A Brief Discussion on the Significance of Chongyang Qi in Sub-health Treatment. Journal of Chengdu University of Traditional Chinese Medicine, 02: 18-19, 38.

[7] Wang QQ, Li K, Zhu L, et al., 2017, Investigation and Analysis of TCM Physique in 528 Patients with Malignant Tumor. Xinjiang Traditional Chinese Medicine, 02: 68-70.

[8] Zhang XN, Wang X, Wang M, et al., 2010, Epidemiological Investigation of TCM Physique Types in 355 Cancer Patients. Chinese Journal of Traditional Chinese Medicine, 5: 13-16.

[9] Zuo WY, Ding HJ, Pu BH, 2017, Identification of Traditional Chinese Medicine Physique in a Community of High Blood Pressure. Shanghai Jilin traditional Chinese Medicine, 1: 36-41.

[10] Huang JY, 2017, A Study on the Investigation and Intervention of Traditional Chinese Medicine Physique in Rural Elderly Experiencers. Chinese Community Physicians, 11: 150-151.

[11] Chen GZ, Zhou YM, Yao ZC, 2016, Investigation and Analysis of Traditional Chinese Medicine Physique of Elderly Community in Longgang District, Shenzhen City China. New Chinese Medicine, 2: 108-112. 
[12] Deng JC, Zheng XW, Chen SH, et al., 2018, Distribution Characteristics of TCM Physique in Slowing Period of Bronchial Asthma. Chinese Journal of Traditional Chinese Medicine, 3: 945-947.

[13] Du YH, Liu HY, Ma WX, et al., 2019, Investigation and Study on TCM Physique Type and TCM Syndromes of 572 Cases of Bronchial Asthma Treated Period. World Journal of Integrated Chinese and Western Medicine, 2: 78-81.

[14] Xu ZY, Wang L, Wang L, et al., 2016, Chinese Medicine Syndrome Study on Diabetes Complicated with Osteoporosis in Yanji Community, Shanghai, China. Journal of Liaoning University of Traditional Chinese Medicine, 11: 100-103.

[15] Wan L, Huang HX, Deng WM, et al., 2019, Investigation and Analysis on Bone Density, TCM Syndrome Type and Influencing Factors of Osteoporosis in Middle-aged and Elderly Women in Guangdong Province. Journal of Osteoporosis in Chinese Medicine, 1: 107-112.

[16] Shen JH, Yang HW, Yang JW, et al., 2016, Investigation on the Correlation between TCM Constitution Type and Infertility Syndrome Type. The Light of Traditional Chinese Medicine, 3: 322-324. 